\title{
Designing of Fermenter and its utilization in food industries
}

Vishwajeet Gaikwad ${ }^{1}$, Anil Panghal ${ }^{1}$, Shubham Jadhav ${ }^{1}$, Poorva Sharma ${ }^{1}$, Akshay Bagal ${ }^{1}$, Akshay Jadhav ${ }^{1}$, Navnidhi Chhikara ${ }^{1 *}$

${ }^{1}$ Department of Food Technology and Nutrition, Lovely Professional University, Punjab

*corresponding author: navnidhi.20759@lpu.co.in

\begin{abstract}
:
Fermenter is a vessel that maintains optimum environment for the development of significant microorganism used in large scale fermentation process and the commercial production of products like Alcoholic beverages, Enzymes, Antibiotics, Organic acids etc. The fermenter aims to produce biological product like vaccines and hormones, it is necessary to monitor and control the different parameters like external and internal mass transfer, heat transfer, fluid velocity, shear stress, agitation speed, aeration rate, cooling rate or heating intensity, and the feeding rate, nutrients, base or acid valve. Fermentation in the fermenter are accomplished in several configuration and these simple configurations are batch, fed-batch and continuous fermentation process. Fermentation process is carried out in small or large size fermenter depending on product quantity. The selection of the suitable process depends on the fermentation kinetics; type of microorganism used and process economic aspects. Improved modelling tools, reactor operation and reactor design in bioreactor is because of mass transfer behaviour and it is important for reaction rate maximising, throughput rates optimization and cost minimizing. The fermenter design, fermentation process, types of the fermenter that are used in industries and heat and mass transfer in fermenter is discussed.
\end{abstract}

Keywords: fermentation, bioreactor, heat transfer, mass transfer

\section{Introduction}

Fermentation process is the oldest technology of all biotechnological process continued from hundreds of years. The study of fermentation process, application and techniques are simply defined as fermentation technology. The fermenter or bioreactor are defined as the heart of fermentation process within this fermenter many reactions and activities occur. In fermentation technology whole study is involve, activities like downstream, upstream and midstream studying and controlling them.Fermentation technology studies needs essential efforts from different disciplines such as bioprocess and chemical engineering, microbiology, biochemistry, genetics and even physics and mathematics. (Jagani et al. 2010) 
The fermenter/bioreactor is the vessel that provides biomechanical and biochemical environment which regulate the transfer of oxygen, nutrient to cells and metabolic products from cell. Bioreactor vessels are designed to utilize activity of catalyst to attain desired chemical transformation (Sharma et al. 2012; Bueno et al. 2004). Bioreactors are also defined as engineered device designed for metabolic activity and optimal growth of organisms with the help of the microorganism, enzyme, biocatalyst and the cells of plants and animal. With fermenter metabolic fluxes are altered and cell resources are diverted to the more anticipated pathways while undesirable one are inhibited. For instance, by commanding a temperature profile given on the culture, certain enzymes are denatured, thus arranging the metabolic routes over others within the cells (Gueguimet al. 2002). Fermenter or Bioreactor are cylindrical shape vessel having hemispherical bottom and top and they are mostly made of glass and stainless steel. Difference between classic composting system and the bioreactor is controlling and measurement of the composting process parameters is more in bioreactor (Jagani et al. 2010). Bioreactors are dissimilar from the conventional chemical reactor to some extent. They support biological entities and control them. Fermenter system should be tough enough to provide greater degree of control over contamination and when the organism are less stable and more sensitive than chemicals (Williams et al. 2002).

The fermenter is involved in processing of beer, wine, vinegar, yoghurt, sauerkraut. The evolution from native art to commercial practice of these products requires improvement in process to increase shelf life and to maintain acceptability and the quality of the product, equipment and the process control parameters remained simple. Adequate heat transfer, mass transfer, substrate in appropriate amount, no under or over dosing of feed in fermenter should maintained. Proper supply of the adequate substrate, salts, oxygen, suspended solids and water availability for aerobic system should be ensured. Care should be taken in gas evolution product and by-product. Some factors regarding to the attributes of fermenter design requirement are scale-up, regulating techniques, typical construction and measuring, sterilization, flexibility in operations, process control devices that are compatible with upstream and downstream processes and antifoaming measures. (Sharma et al. 2012).

Aseptic environment is well defined as defense against entry of the unwanted microorganism and contamination is preventing escape of viable cell form the process. Fermenter system provide both type of the environment where ever they are mandatory. Contamination is related in all of the process of fermentation but containment is essential after the pathogenic organism occurrence in the fermentation practice. (Gudinet al. 1991) stated that condition of the fermenter for living organism should be favorable to display their activity in 
defined condition. This calls for a series of distinct attributes in the engineering reaction of bio catalytic processes. Many times Bioreactor and fermenter term are used synonymously. The slight difference between these two is fermenter is used for microbial culture and bioreactor is used for mass culture of animal and plant cells.

\section{Fermenter Design and Operation}

The fermenter design should provide high quality product in low cost with improved productivity and authentication of the desired parameters. The design of the fermenter and mode of operation relies upon microorganism production, product value, condition necessary for the desired product formation and production scale. The effective fermenter provides positive influence on the biological reaction and prevents foreign contamination. In fermenter capital investment and operating cost are important factors that to be considered. (Eibl et al. 2008) reported that during the fermentation process proper mixing, uniform shear rates, mono septic conditions should be maintained. Aeration of the culture is achieved by one of the following methods: increasing atmospheric pressure or enhancing the restricted pressure of oxygen and direct sparging, indirect and/or membrane aeration or by medium perfusion. (Naraendranathanet al.1998) discussed about characteristics of a good bioreactor design which include: a simple, economical and robust mechanical design, beneath aseptic conditions easy operation, flexibility in the process, nonappearance of dead zones and transfer of heat and mass. The bioreactor design must control $\mathrm{pH}$, temperature, oxygen tension, allow aseptic operation and the shear stress. The system should also allow for automated processing steps. This all is essential not only to control, but also for future routine manufacturing (Martin et al.2004).

The biotechnological process has several aspects, which needs special care in bioreactor designing. The cell growth, reaction rate and process strength in the bioreactor depends upon environmental condition. Condition in bioreactor like $\mathrm{pH}$, temperature, agitation speed, dissolved oxygen level, foam production, gas low rates etc. necessary to control and closely monitored (Chen et al. 2006).

\section{Size of Fermenter Vessel and Material}

The sizes of the bioreactor vary widely from the microbial cell $\left(\right.$ fewmm $\left.{ }^{3}\right)$ to shake flask $(100-1000 \mathrm{ml})$ to laboratory scale vessel $(1-50 \mathrm{~L})$ to pilot level $\left(0.3-10 \mathrm{~m}^{3}\right)$ to industrial scale $\left(2-500 \mathrm{~m}^{3}\right)$ for large volume industrial applications. The width of the manufacturing substance increases with scale. At 3,00,000-4,00,000 $\mathrm{dm}^{3}$ capacity, $7 \mathrm{~mm}$ plate are generally utilized for 
the side of the vessel and $10 \mathrm{~mm}$ plate for the upper and lower part, it should be hemispherical to resist pressures.

The material used for the fabrication of the fermenter are discussed by (Kaushik et al. 2014). Lab scale fermenter are typically constructed with the glass and the pilot scale and the industrial scale vessel are normally fabricated with stainless steel or at they should have cladding of the stainless steel. The presence of a thin hydrous oxide film on the exterior of the steel reduces the corrosion of stainless steel. The configuration of film differs with industrial process treatment and altered steel alloys. Chromium is used to alleviate the film and considered as insoluble, nonporous and self-healing. When exposed to environment or oxidising agent damaged film will be repair itself. (Duurkoop et al. 1992) suggested that in chromium steel high percentage of nickel improves engineering properties and enhances their resistance. The resistance of stainless steels is improved by the existence of molybdenum to the mixture of halogen salts and opposing by chloride ions in brine or sea water. Nowadays molybdenum commonly used in the construction of the fermenter containing $10 \%$ nickel and $18 \%$ chromium. Wood, plastic and concentrate are used where no chance of contamination in the system.

\section{Basic Feature of Fermenter Design:}

The basic feature of bioreactor which include agitation system, foam control, oxygen delivering system, headspace volume, sampling port, temperature and $\mathrm{pH}$ control system, sterilization system and lines for charging \& emptying the reactor (Alaghlavi et al. 2013). These are described in Table 1.

\subsection{Aeration and Agitation system}

\subsubsection{Aeration system}

Aeration supports the growth of the organism by providing appropriate amount of oxygen in the fermenter. The device such as sparger is used to introduce the air in the fermenter. Aerators producing fine bubble should be used. Oxygen is easily transfer through the sparger to greater amount with the large bubbles which has less surface area than the smaller bubbles. In the case of airlift fermenter agitationis not necessary, aeration provides enough agitation. But this is feasible with medium or low viscosity or less amount of total solids. For providing agitation with the help of aeration, the height/ diameter ratio of the fermenter should be 5:1. Sparger contains air filter to supply sterile air into the fermenter.

Three types of the sparger are used widely (a) porous sparger (b) orifice sparger (c) nozzle sparger 


\subsubsection{Agitation system}

Agitation offers uniform mixing of the cells in standardized nutrient medium. Agitation offers mass fluid and gas phase mixing, ease of oxygen transfer, dispersion of air and transfer of heat and maintains uniform atmosphere in the fermenter. Agitation in the fermenter is achieved with agitator. Four different classes of the agitator (impeller) are Marine impeller, Vaned disc, Disc turbine and Open turbine of variable pitch.

Flooding in the fermenter by air bubbles is prevented by the Disc turbine. The conditions of the flooding is specified when the mass flow pattern in the fermenter is generally related with agitator design (radial with the Rushton turbine)is misplaced and changed by a centrally flowing air-broth plume up in the middle of the fermenter with a liquid flow as an annulus ( Nienow et al. 2014).

Table 1. Different parts of Fermenter and its function (Jagani et al. 2010; Kaushik et al. 2014)

\begin{tabular}{|l|l|}
\hline Parts of Fermenter & Function \\
\hline Impellor (agitator) & $\begin{array}{l}\text { Continuous stirring of media, distribution of the oxygen } \\
\text { throughout the system and prevent cells from settling down }\end{array}$ \\
\hline Sparger (Aerator) & $\begin{array}{l}\text { Introduce sterile air or oxygen to the media in the aerobic } \\
\text { fermentation process }\end{array}$ \\
\hline Baffles (vortex breaker) & Provides better mixing by disrupting the vortex formation \\
\hline Temperature probe & $\begin{array}{l}\text { Measure and monitor temperature change in the medium during } \\
\text { the fermentation process }\end{array}$ \\
\hline Cooling Jacket & maintains the temperature of process \\
\hline pH probe & Evaluate and monitor the change in pH of the medium \\
\hline Level probe & Measure the level of medium \\
\hline Foam probe & Detect the presence of the foam \\
\hline Acid & Neutralizes the basic environment and maintain the pH \\
\hline Base & $\begin{array}{l}\text { Neutralizes the acidic environment and maintains the required } \\
\text { pH }\end{array}$ \\
\hline Antifoam & Breakdown and avoid foam occurrence in the medium \\
\hline Sampling point & Ro acquire samples throughout the process \\
\hline
\end{tabular}




\begin{tabular}{|l|l|}
\hline Control panel & Monitor all parameters in the system \\
\hline Inlet air filter & Filtration of air before entering the fermenter \\
\hline Exhaust air filter & Prevention of contaminants from escaping \\
\hline Rotameter & Measure flow rate of liquid or air \\
\hline Dissolve oxygen probe & Measure dissolve oxygen in the fermenter \\
\hline Pressure gauge & Measure internal pressure of the fermenter \\
\hline
\end{tabular}

\section{Fermenter Operation}

There are three different types of operation that are batch, continuous and fed-batch or semi-continuous depending on feed of the fermentation media and the cultures into bioreactor (McNeil and Harvey 2008). The relationship of the feeding of substrate to the biomass and yields suggest the excellent fermentation mode (Raj and Karanth2006). For the centuries Traditional batch stirred tank reactors (STRs) and continuously stirred tank reactors (CSTRs) are existed. Due to their simplicity they are commonly used in bioprocessing industries (McNeil and Harvey 2008). Other bioreactor having different design and operational attributes are membrane bioreactor, drum reactors, photo-bioreactors, fluidized bed bioreactors, air lift bioreactors and bubble column reactor. These all are developed to provide various application in specific processes.

\subsection{Batch Process}

Batch fermentation is a closed culture technique in which the small quantity of nutrient is inoculated with the variety of organism to let the fermentation. Batch fermentation is the traditional process, during fermentation process no ingredient are added after inoculation, except base or acid in controlling $\mathrm{pH}$. In this fermentation process, organism effects in high substrate concentration primarily and a high product concentration lastly. (Williams et al.2002) observed that for the promotion of aerobic cultivation in fermenter, the aeration of medium with sterile air to provide constant flow of oxygen. Gaseous by-product such as carbon dioxide are removed, gas removal processes and the aeration takes place in this process.

\subsubsection{Batch Fermenter Design}

The impellers in STRs connected to an exterior motor, which runs the stirrer system. The assembly of agitator including seal is a way of contamination so the shaft has to pass from aseptic seals into bioreactor (Abbott et al. 2013). (Garcia-Ochoa and Gomez 2009; Martin et 
al.2008) reported about contribution of the impellers in the uniform mixing and the termination of the essential atmospheric oxygen into aqueous phase.

The efficiency of the agitation is determined by the shape of impeller blades and speed of agitation. The mass transfer rates and mixing are affected due to variety of stirrer, stirrer speed and the rate of gas flow used. Batch type of fermenter are preferred for high value end product and low volume, particularly to obtain product yield. Such type of fermenter are used when more than one products are produced prepared in same equipment or in the case of highly viscous liquid where continuous flow is difficult. (Williamet al. 2002). Batch type fermenter are more flexible with varying product system, they low down the risk of contamination and the investment is also low as compared to the continuous processes for same volume.

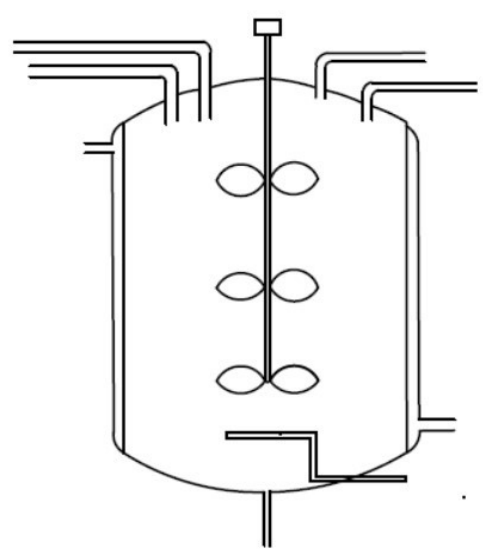

Figure 1. Stirrer tank (Jagani et al. 2010)

\subsection{Continuous Process}

In the continuous process of the fermenter new medium is constantly added in the system along with that continuously removal of the product from the bioreactor. (Abbott et al. 2013). This supports the cells in growth phase and keeps the capacity of reactor constant. Thus, steady state is attained, the connection between microorganism behaviour is established depending upon the availability conditions of the nutrient. The continuous product are formed with higher yield. In continuous stirred-tank, the reactants are very well mixed. Other name for the reactor is back mix reactor.Continuous feeding process is the specific feature of continuous bioreactor. (Martin and Montes 2008) suggested that hydraulic or mechanical agitation is 
required to accomplish uniform temperature and composition. To maintain steady state, the culture is continuously fed to medium in the bioreactor. The control parameters and the reaction variables remain consistent, within bioreactor it establish a time perpetual state. This result in the continuous output (McNeil and Harvey 2008). (Kaushiket al. 2014) suggested that by automating the process, time could be saved in filling, emptying, and sterilizing the bioreactor with reduced toxicity risks and reduce labour cost. CSTRs can yield high product quality, due to presence of the mechanical pumps CSTRs consume more energy.

\subsubsection{Continuous Stirred Tank Reactor (CSTR)}

In the CSTR, with one or extra agitator, fermentation broth is continuously stirred and the composition is identical and homogeneous to that for outgoing flow into the reactor. Such type of reactor are usually operated in syngas fermentation. Application of the CSTR is constant flow of the gas throughout the liquid that normally contains a diluted solution of basic nutrients for the organism to develop and survive on. (Tang et al.2010) has evaluated the performance of reactor in concurrent and ethanol fermentation in addition with flocculating yeast strain. Using gas stripping the product removed from fermentation media also reported with the Continuous stirred tank reactor (Liu et al. 1990).

\subsubsection{Plug Flow Reactor (PFR)}

In the plug flow reactor (PFR), the reactants are driven via tube or pipe with a constant velocity profile across theradius, and the reaction continues as the reagents moves within thePFR, with dispersion expected to be small in the axial direction (Anandaet al. 2014).

In PFR operation, inoculum is continuously fed to the reactor.
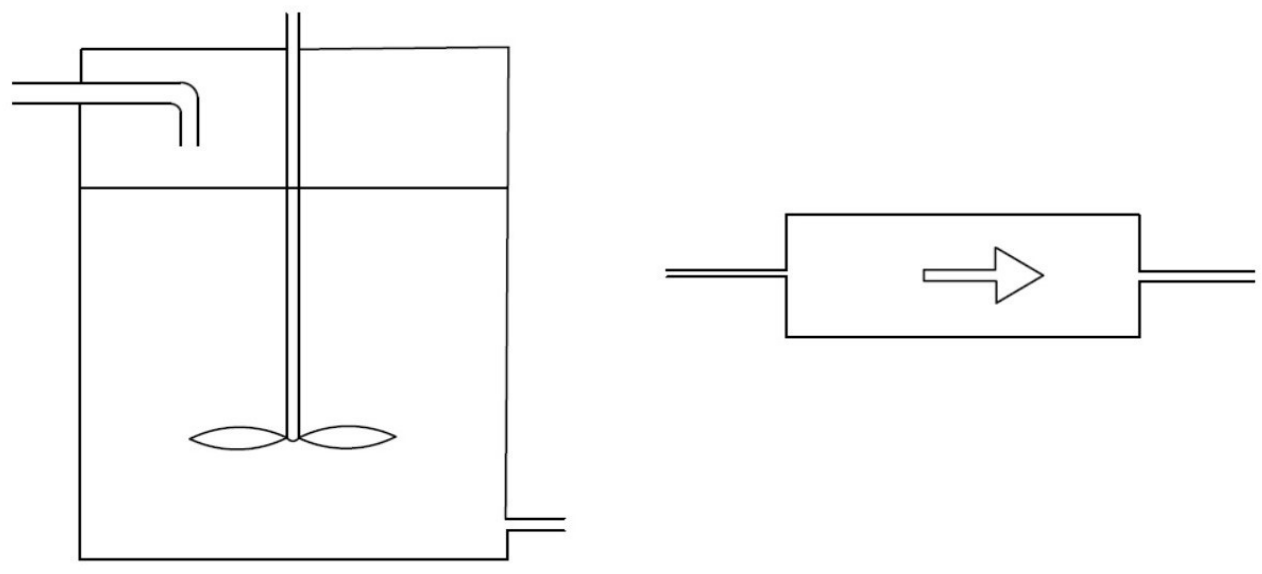

(a)

(b) 
Figure 2.(a) Stirred tank reactor(CSTR) (b)Plug flow reactor (PFR). (Ananda et al. 2014)

\subsection{Fed Batch or Semi-Continuous Process}

Fed-batch fermentation process is a system in the middle of batch fermentation and continuous fermentation.During whole fermentation process in Fed batch or semi-continuous process, broth is taken off from the reactor, however the rate of nutrient are limited thathelps in increasing yield of product and controlling reaction rate. In this process sterilized condition are maintained during the operation and the yield is collected at the end of the process as in batch bioreactor (Abbott et al. 2013). Such type of vessel perform safer and stable operation in the batch bioreactor. An elaborate sequences of equipment is necessary to maintain suitable feed rates with the accurate constituent in the fed-batch reactors.However, the fed-batch methodhas several advantages than batch and continuous process.

\subsubsection{The Constant Volume or Fixed Volume Fed-Batch Reactors}

In this type of fed-batch reactor, the substrate is provided in the limited amount without diluting a culture. The growth limiting substance are fed in undiluted form, e.g. solid or concentrated liquid volume of culture can be maintained constant. Alternatively, addition of substrate by dialysis withoutdisturbing the culture volume can be done. (Anandaet al. 2014).A periodic removal of a part of the culture is mandatory in a fix cyclic fed batch reactor.

\subsubsection{Thevariable volume fed-batch reactors}

In the variable volume fed-batch reactor, change in size occurs with fermentation time due to substrate feed. Volume change can be controlled depended on the need such as microorganism characteristics and fermentation kinetics. In this reactor, liquid volumes are used as a variables in optimizing the product yield. For example, at certain stage the culture is replaced from the system with fresh medium where the fermentation process is not effective anymore. The reduction of volume in the process results in rise in the specific growth rate, followed by a steady fall as the quasi-steady state is created. 


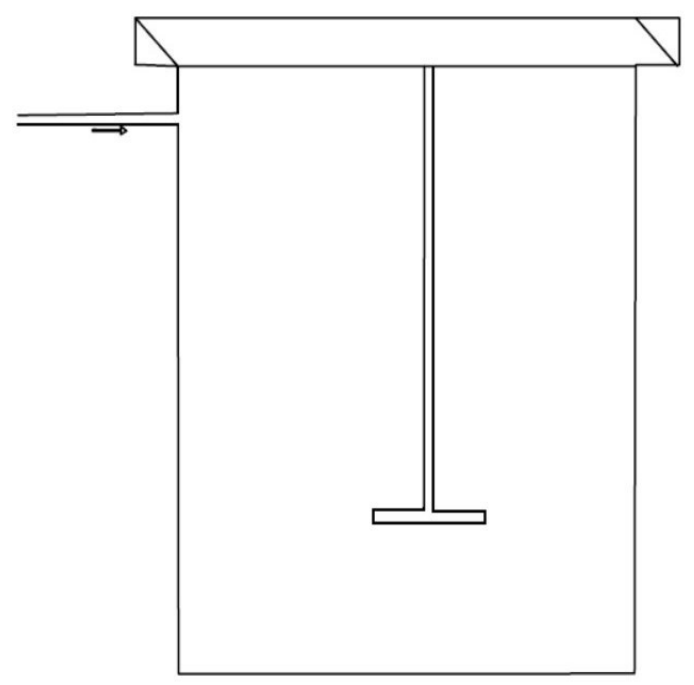

Figure 3. Fed-batch fermenter (Abbott et al. 2013)

\section{Types of Fermenter}

\subsection{Stirred Tank Fermenter}

Stirrer tank fermenter (STF) are known for their flexibility so they one of the most frequently used fermenter. (Chisti et al.2010). STF are cylindrical in shape and in STF motors drives the shaft and stir the content in the tank. Depending on the scale of process and other aspect agitator might be bottom driven or top driven. Commonly used are top entry-stirrer (agitator) model, it has so many benefits such as robustness, reliability and easily operated and rarely used are the bottom entry-stirrer (agitator) model. The agitator might be top driven or bottom. The shaft support more than two impellers positioned almost one impeller-diameter separately (Chisti et al.2010). The characteristic decision variables are: size, type, impellers and sparger size. This type of reactor has following functions: aeration of liquid, solids suspension, homogenization, gas-liquid mixture dispersion and exchange of the heat.

Laboratory-scale stirrer tank fermenter are fabricated with the borosilicate glass and the stainless steel lid. Capacity of these fermenters is 1 to $100 \mathrm{~L}$. Fermenter with stainless steel has special requirement in the laboratories. (Ali et al.2002) has studied about laboratory-scale stirred fermenter that was designed for production of citric acid using AspergillusnigerGCBT7 having capacity of the $15 \mathrm{~L}$ with working capacity of $9 \mathrm{~L}$. (Vamanu et al. 2009) reported the condition of fermenter that properly mixes the medium for 15 min. Such type of tanks suffer 
because of their low productivity. The Stirred tank reactor's provides tremendous mass transfer and advantageous mixing. Attrition problem occurs in the vessel with the immobilized cells, however by splitting the zone of mixing one can easily and effectively function the system.

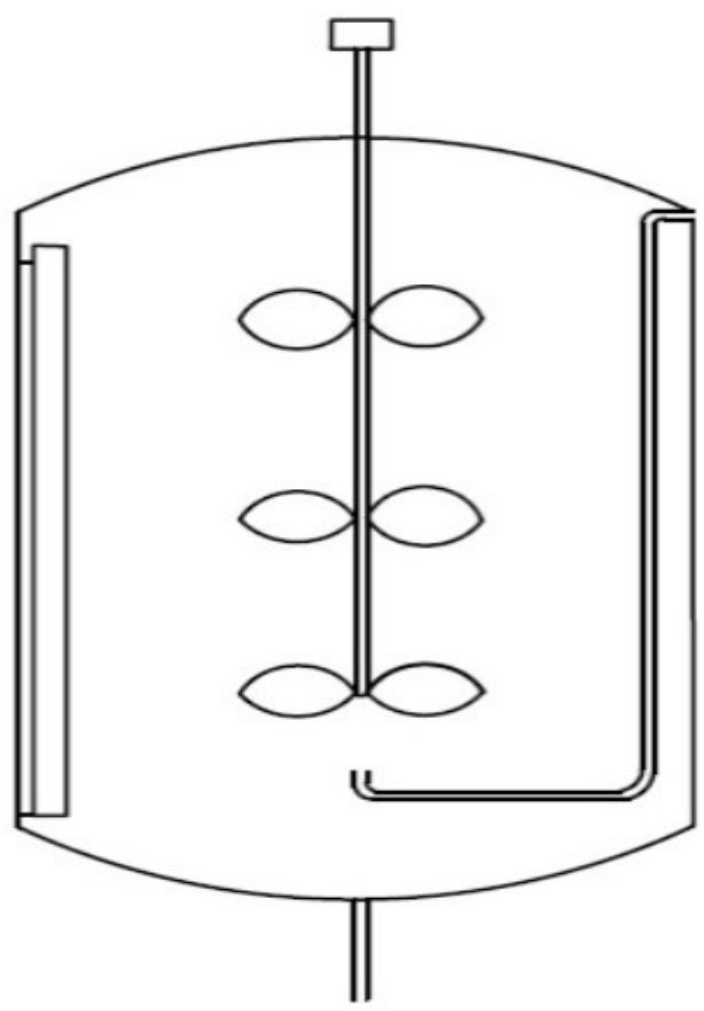

Figure 4. Stirred tank (Jagani et al. 2010)

\subsection{Airlift Fermenter}

Airlift fermenter is also recognized as tower reactor. An airlift fermenter vessel is cylindrical in shape in which air is presented at the bottom of receptacle which arises through the column to the medium. Operational proportion of the airlift fermenter is more than $6: 1$ (height /weight). ALFs capable of using for free or together with immobilized cells that are suitable for bacteria, yeast, fungi and animal and plant cells. These type of the fermenter does not have agitation system, but contents in the vessel is agitated with the air introduced from the bottom. The cell mixture flows all over the place in the column as a significance of the gradient of air bubbles in the various sections of the fermenter. The fluid of volume is separated within two connected regions by means of draft tube or baffles for improving circulation and oxygen transmission and aligning shear forces in the apparatus (Veera and Joshi 2001). From the two 
zones only one zone is sparged with the gas or air. Zone that is not sparged is known as downcomer, and the region which receives gas is the receiver (Chisti and Moo-Young 2001). These come in two model i.e. external loop design and the internal loop design. Riser and the downcomer enclosed in similar shell in the internal-loop and in the external- loop downcomer and riser are distinct tubes interconnected near bottom and the top (Chistiet al. 1999).

In the production of citric acid, airlift fermenter was used with $900 \mathrm{~m}^{3}$ volume using Aspergillus niger. The airlifts Fermenter are fabricated with the stainless steel to resist the acidity because ordinary steel dissolve at $\mathrm{pH} 1-2$. Airlift fermenter is generally suitable for the aerobic organism due to the oxygen mass transmission coefficient is higher than stirred tank reactors. This fermenter is perfect for the creation of SCP as carbon substrate from methanol. Generation of the excess heat during agitation is avoided in this fermenter. The knowledge of producing SCP from methanol procedure related to Imperial Chemical Industries (ICI, UK).

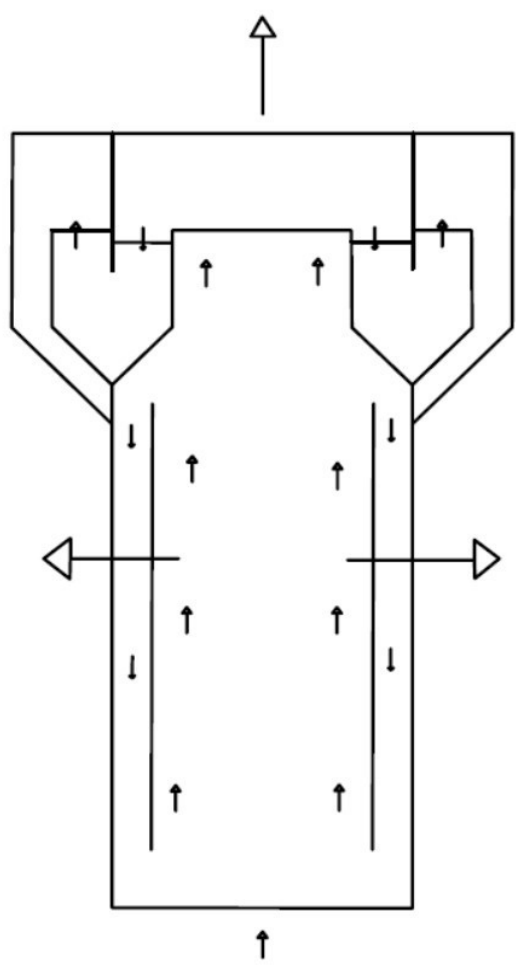

Figure 5. Airlift reactor (Siegel and Robinson 1992).

\subsection{Bubble Column Reactor}

The reactor is one of the simplest type of the reactor and it is easy to scale up (Kantarci et al.2005). A bubble column reactor is generally cylindrical shape receptacle which contain gas provider at the bottom side. Helmut Gerstenberg et al. applied it for first time. (Borakb and 
Kutlu 2005) reported that in bubble column reactor gas is sparged in the manner of bubbles into liquid-solid suspension or liquefied phase. The existence of solid phase in the vessel commonly mentioned as slurry bubble column reactors. The mixing in the reactor is achieved by gas sparging also it needs very small amount energy than the automated stirring (Nigar et al. 2005). Comparing with other reactor having agitator bubbles make less shear stress (Kantarci et al.2005). Bubble column reactor has many advantages as comparing with other type of reactors. These types of reactor requires less maintenance and have good heat and mass transfer attributes, operating cost is less because of their compactness along with stability of the catalyst.

Bubble column reactors are utilized by many industries such as biochemical, petrochemical, and metallurgical and chemical industries (Degaleesan et al. 2001). Use of these reactor especially in the production of artificial fuels by transformation of the gas process, chemical processing such as chlorination, polymerization, alkylation, and hydrogenation and in biochemical process for example biological waste water management and fermentation (Prakash et al.2001). Accessible chemical agent addition and taking out ability and plug-free process are additional benefits that reduce bubble columns an appealing reactor choice (Prakash et al. 2001). (Ogbonnaet al.2001) study was depending on ethanol fuel production from sugar-beet juice in bubble column reactor. Yeast cells (Saccharomyces cerevisiae) were used so as to examine the possibility of the procedure. 


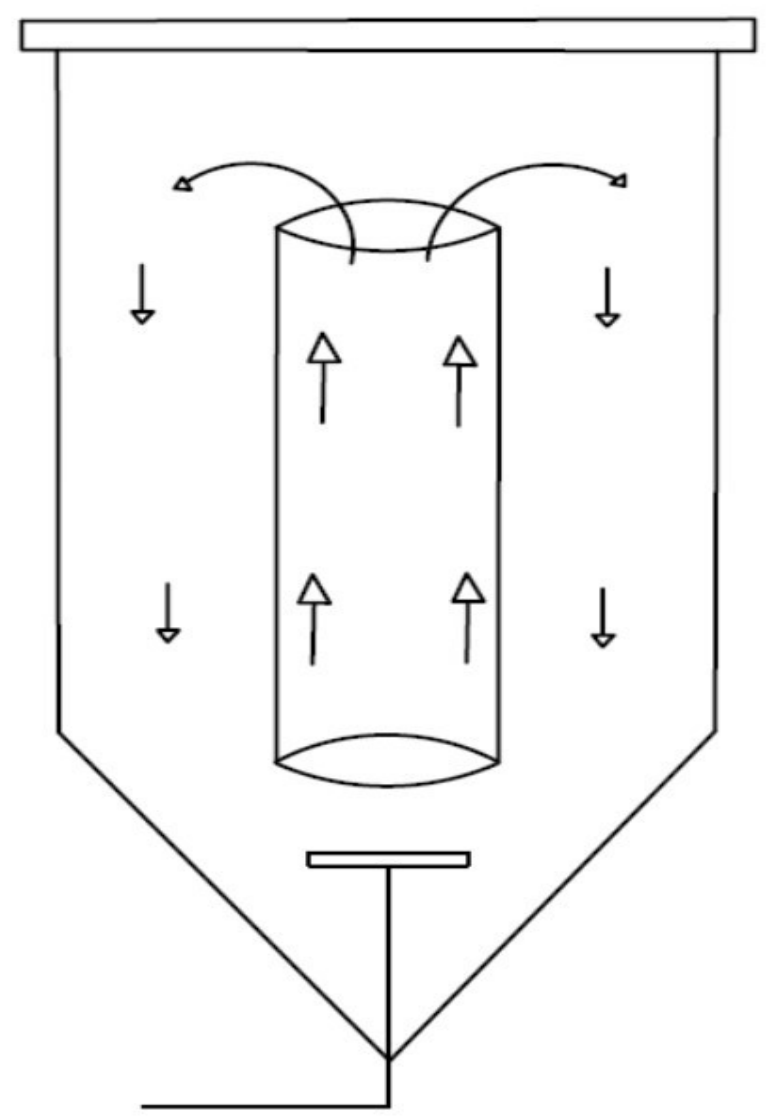

Figure 6. Bubble column reactor (Wilkinson et al. 1992).

\subsection{Fluidized Bed Reactor}

FBB has many advantages over other type of reactor so in current years use of this type of reactor has increased. Fluidized bed reactor are developed for the biological system has cells as biocatalyst that are three phase system (liquid, solid and gas). This type of reactor contains packed bed with small size particles which moves the fluid. The minor particle size accelerates higher rate of oxygen transfer, mass transfer, higher mixing rates and nutrients to the cells. Mostly the constituents used in FBBs are of three types: (a) inert core on which the biomass is produced by cell attachment. (b) Porous particles in which the biocatalyst is entrapped. (c) Cell aggregates/ flocs (self-immobilization). Some problems occurred in the packed bed reactor such as high liquid pressure drop, channelling, clogging, compaction of bed is completely prevented in fluidized bed reactor. 
Operation is in continuous state with temperature gradient and uniform particle mixing. FBB are same as bubble column reactor with expanded cross section area at the top of the vessel so as to recirculate the liquid that is constantly pumped from the bottom of the receptacle at a velocity that is satisfactory to preserve them in suspension (Chistietal.2010). Agitation of the medium is supported with the action of the pump in this bioreactor. FBB is one of the method for sustaining high biomass concentration and excellent mass transfer in continuous process. The concentration of the biocatalyst is significantly greater and washout limitations of free cell systems is affected (Gibilaro et al.2001). FBBs provides lesser attrition of solid particle than the conventional mechanical stirrer reactor and also volumetric productivity is higher than the packed bed reactor. FBBs is widely use in bioprocess improvement. The efficiency of fluidized bed reactor depends on the attachment of particles that are maintained in suspension by an upward flow rate of the fluid to be treated. These particles are also called as biofilm carrier either they are inert core on which the biomass is produced by cell attachment; or porous particles in which the biocatalyst is trapped (self-immobilization).

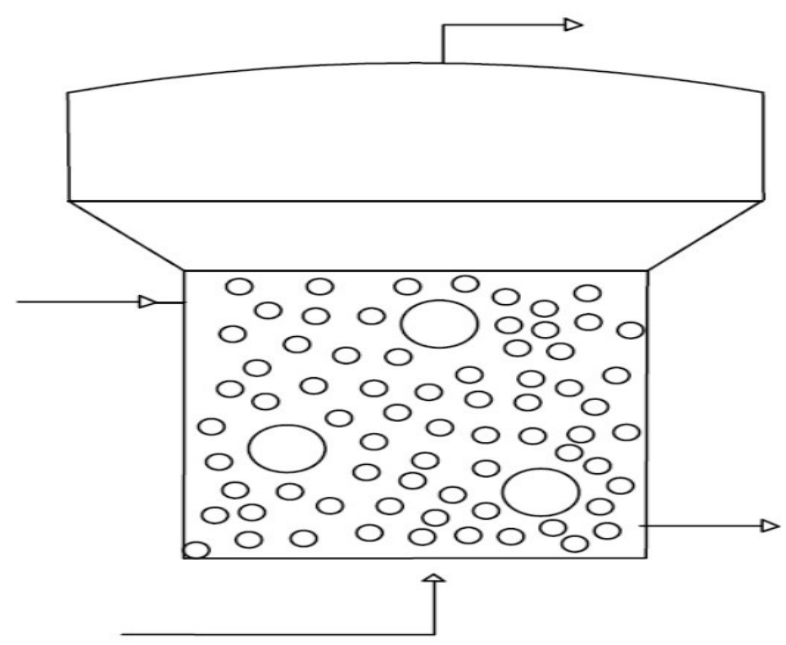

Figure 7. Fluidized bed reactor (Kwong et al. 2000).

\subsection{Packed Bed Reactor}

Packed bed bioreactor is well known as fixed bed bioreactor. It is normally used in wastewater engineering with biofilms attached. The use of packed reactor gains significance after whole cell immobilization technique when it is demonstrated. The nutrients are fed from 
the top or bottom with immobilized biocatalyst packed in the column. The packed bed reactor are commonly treated with the immobilized cells. The reactor constitute a bed of packing, made with glass, ceramic, polymer, natural material and they exist in various size and shapes which allows the flow of fluid from one end to other end. Packed bioreactor can run in the dribble flow mode or in the submerged mode with aeration. The flow rate in the channelscan be increase to remove external mass transfer limitation in the adjacentliquid film. The rate of high pressure drops, at the same time, plugging can be avoided in the reactor (Wang et al. 1992). During operation, due to modifications in the bed porosity variation in the flow characteristics arises in the packed bed bioreactor.

Packed bed belongs to the group of plug flow reactor where backmixing is not present, but in this reactor slight backmixing arises which affects the attributes of the fermentation. They are normally used as soon as substrate inhibition administers the rate of reaction Several modification are made to the reactor such as tapered bed are used to decrease the pressure drop, horizontal bed, inclined bed, rotary horizontal reactors have been tested with partial achievement.

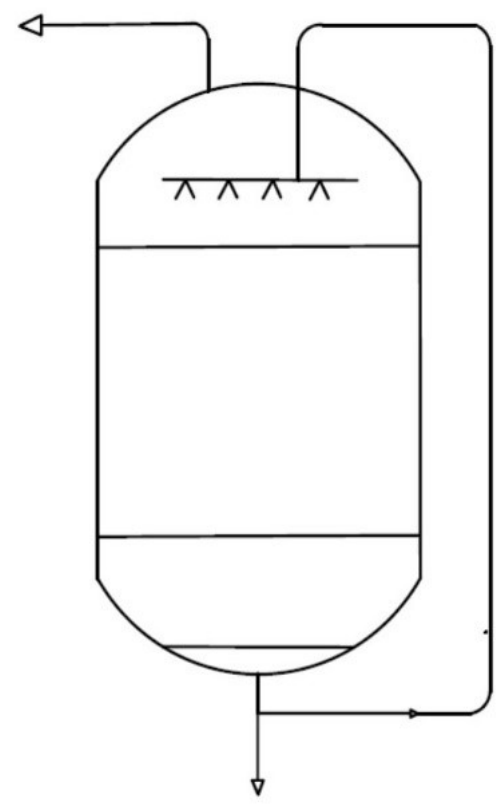

Figure 8.Packed bed reactor (Siegel and Robinson 1992) 
Membrane bioreactor contains hollow fibre system which is developed for the immobilization of the yeast, enzymes and bacteria. The hollow fibres are prepared with cellulose acetate with a consistent wall matrix or from polysulfone fibres or acrylic copolymer with irregular wall configuration (Raj and Karanth 2006). Advantages of hollow-fibre reactor for microbial system are biocatalyst regeneration and immediate separation of biomass and the product. Major drawbacks of the reactor are, they are difficult to control and monitor the growth and breakdown of the culture and excessive development of microorganism because of the less oxygen transfer rates. The metabolic activity of cells is inhibited by accumulation of the toxic products in the hollow fibre. In various microbial cell production membrane bioreactors has been widely used (Chang and Furusaki1991). Some investigation are reported on the production of the probiotic cells in membrane reactor, such type of system supports volumetric productivities and high cells yield.

\section{Heat Transfer}

Heat transfer is the transfer of heat from one location to another. Transfer of heat is one of the significant parameter in maintaining and controlling the temperature during various operation in the fermentation process. Generation of heat in the process in fermenter because of the mechanical agitation and metabolic activity of microorganisms and by bubbles formed by the gas, bubbles size increases when passed from the air sparger to the headspace of the fermenter. The work performed by gas expansion by mechanically agitated system is usually very less of the total. For most fermentation process heat is removed by cooling. The fermentation process run above ambient temperature that involves thermophile organisms, for such process involvement of heat is needed. Heat transfer is mainlyattained by internal coils or via outer jacket surrounding internal phase in fermenter. Heat is always conducted through the coils, baffles or vessel wall, no direct contact exist between fermentation medium and the heating/cooling system. These type of systems are also applied to sterilize the fermenter, by injection of the pasteurized steam.(Jackson et. al. 1990) reported that heat generated by the fermentation is difficult to remove when the superficial area enclosed by the heating jacket become insignificant. In this situation with the help of internal loops accurate temperature can achieve and the circulation of cold water is needed in the area. (Atkinson and Mavituna1991) have provided a technique to determine the loss of heat because of evaporation ( $Q$ evap), a formula to determine the sensible heat loss for flow streams ( $Q$ sen $)$ data to estimate $(Q m e t)$ for a range of substrates and techniques to determine power input for $(Q \mathrm{ag})$ and $Q$ gas. 
Transfer of heat between the vessel surface and the microorganism is slightly comparable to that of mass transfer, involves alteration between bulk fluid as well as the cells and between cooling surface and bulk fluid. The temperature in the mass fluid is calculated by the dynamic balance between the process of evolution of heat by cells and agitator and removal of heat from the container wall in case of mass transfer.As compared to mass-transfer coefficients determination, the cell-to-fluid heat-transfer coefficients is further challenging, if the mass of the cell aggregates expands, at same time heat accumulation within cell aggregates at a certainstage of metabolic activity also increases. In the given temperature of bulk fluid, the average temperature of cell rises with increasing aggregation. Effects of the biological activity is fouling, it occurs on the heat-transfer surfaces, either by the cell deposition or material attack in solution. Overall heat-transfer coefficient is reduced by such fouling. The design, material, position and reinforcing of the coil should be in such a way that the deposition risk will minimize by confirming free flow of the suspension on the exterior of the coils and increase in heat transfer co-efficient.

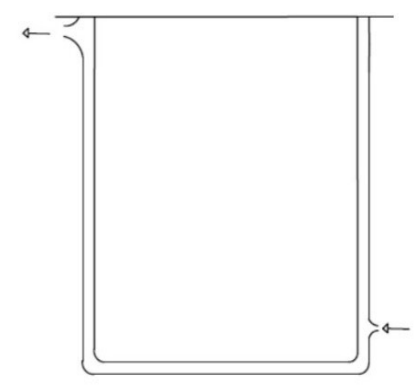

(a)

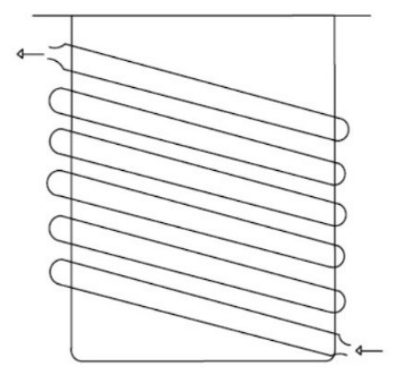

(b)

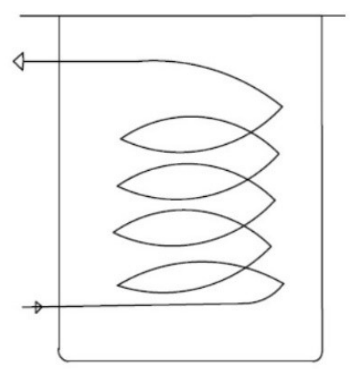

(c) 


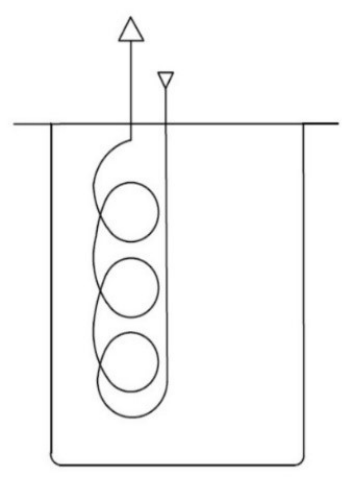

(d)

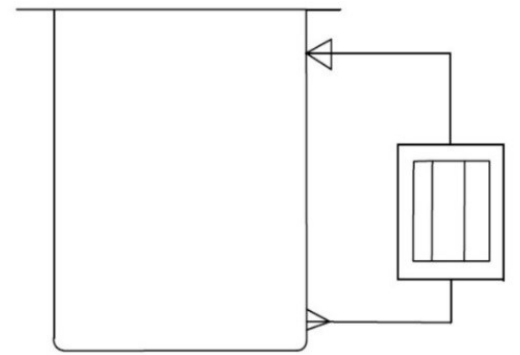

(e)

Figure 9. Heat transfer configuration for fermenter are (a) jacketed vessel (b) external coils (c) internal helical coils (d) internal baffle type coils (e) external heat exchanger (Pauline Met al. 1995).

\section{Mass transfer}

Transfer of mass from one location to the other location under the effect of concentration gradient and concentration difference in the system. Mass transfer within the process is important to the entireoperation. Basically, two aspects to the mass transfer process are -the less or more uniform supply of the product molecules and the substrate in the bulk of fluid and the transfer between microbial cells and the bulk of fluid. Transfer of mass occurs between two phases, liquid-liquid and gas-liquid. In gas -liquid reactions the mechanically agitated stirred tanks are mostly used, waste treatment and the aerobic fermentation. These type of vessel provides good mixing and has mass of high values and heat transfer coefficients. The mass transfer coefficient, $k \mathrm{~L} a$ is the most significant parameter effecting design and operation of unit. (Lau et al. 2012) has evaluated the mass transfer coefficient by using oxygen sorption method. Mass transfer in the gas -liquid reaction system is studied by many investigator. (Lauand Tao Chen 2012) reported that interfacial mass transfer is derived on the basis of its bubble size distribution. The mass transfer coefficient of liquid side can calculated from the interfacial mass transfer area and volumetric mass transfer coefficient.

Overall mass transfer coefficient is a combination of mass transfer coefficients of gas phase, liquid phase and biofilm as given in the following equation

$$
1 / \text { koverall }=1 / \mathrm{k}_{\mathrm{G}}+1 / \mathrm{k}_{\mathrm{L}}+1 / \mathrm{k}_{\mathrm{B}}
$$


Where,

$\mathrm{K}_{\text {overall }}$ - Overall mass transfer coefficient

$\mathrm{k}_{\mathrm{G}}-$ Mass transfer rate coefficient of gas phase

$\mathrm{k}_{\mathrm{L}}$ - Mass transfer rate coefficient of liquid phase

$\mathrm{k}_{\mathrm{B}}$ - Mass transfer rate coefficient of the biofilm

Mass transfer of liquid-side alters with gas velocity controlled by varying bubble rising parameters. Mass transfer rate of gas-liquid state is improved by static liquid height.Efficient gas-liquid contacting has great importance to numerous industrial applications, together with biological gas treatment. When mass transfer is limited, the metabolic rate of the microorganism reduces and the organisms may reactundesirably resulting stress (Lebreroetal. 2010).A large amount of relationships are available for the volumetric mass transfer coefficient, but often the results from various equations are not in contract with others (GarciaOchoa and Gomez 1998). So they are being expatriated by theoretical or predictive models, based on more significant principles.Dynamic measurement method is used to compute the volumetric mass transfer coefficient, $\mathrm{kLa}$, assuming no depletion order for gas phase and proper mixing in liquid phase. (Salehiet al. 2013) reported that in bioreactor transport of dissolved oxygen occurs in three different phases (i) bulk fluid phase of the bioreactor (global mass transfer), (ii) from the bulk to the superficial of the aggregated cells (internal mass transfer), (iii) throughout the aggregated cells (external mass transfer).The internal mass transfer rates relies on a combination of convection mechanism, porosity and scaffold's structure, diffusion rates and the external mass transfer relies on bioreactor's hydrodynamic condition. For minimizing costs and maximizing the efficiency, it is necessary to understand the behaviour of mass transfer for gas treatment in fermenter resulting improved modelling tools and more advanced reactor operations (Rolfe et al. 2006).

\section{Scale up in Fermenter}

Scale-up is the rise in level or dimensions of fermenter, e.g. laboratory scale to pilot scale or from pilot scale to large production scale. The key feature to be measured on the scaleup is that the volume of the fermenter increased by the cubed function and the surface area is increased by the square function so air flow rate, carbon dioxide and oxygen transfer, agitation rate and foaming will be affected. Scale-up and scale-down in relation with agitation and aeration is discussed by (Junkeret al. 2004; Junker et al. 2009) in detail. If some parameters such as impeller speed remains perpetual at the time of fermentation process as power output, it increases to an extremely large, and becomes economically unviable. Thus it is difficult to 
regulate the same level of mixing on scale-up as it leads to less favourable mixing behaviour (Schmidt et al. 2005).

Scale-up can also alter the generation of foam, shear forces and rate of elimination of carbon dioxide. Scaling-up using continuous oxygen transfer rate (OTR) is the ability to calculate or evaluate the volumetric mass transfer coefficient, $\mathrm{kLa}$. It is unlikely to reproduce particular conditions on all levels of operation no matter whatever standards are used in the process. One set of principles are widely used to maintain the similarity of the three different parameters that are power input per unit volume, geometry and superficial air velocity. The outcome of the latter is to decrease the relative volumetric air-flow rate as scale increases.

\section{Conclusion}

Fermenters are used to produce wide range of biological and high value product. They provide the prospect to control and monitor the conditions of the fermentation process with added benefits. Fermenter configuration influences the production rate of the fermentation, several factors like size of fermenter, vessel shape, agitation, aeration, baffles, etc. plays important role in the production. There is no single fermenter which can achieve all the conditions. Different methods are developed to increase the production besides different controlling probes are developed to improve the productivity.

Appropriate calculation methods has been developed to provide quantitative understanding of heat transfer and mass transfer. Innovative technologies are established in different types of fermenter to modify the efficiency rate.

\section{References}

1. Chandrashekhar, H.; Rao J.V. An Overview of fermenter and the design considerations to enhance its productivity. Pharmacologyonline. 2010, 1, 261-301.

2. Sharma, K.R. Design and operational key factors of bioreactor. Biotech Articles. 2012.

3. Bueno, E.M.; Bilgen, B.; Carrier, R.L.; Barabino, G.A. Increased rate of chondrocyte aggregation in a wavy-walled bioreactor. Biotechnology and bioengineering. 2004, 88, 767-77.

4. Lateef, A.; Kana, M.Z.; EB, G.K.; J.K, O. Constructional features of a 15-litre homemade bioreactor for fed-batch fermentations. African Journal of Biotechnology. 2003, 2, 233-6.

5. Williams, J.A. Keys to Bioreactor Selection, CEP Magazine. Google Scholar. 2007, 2002, 34-41.

6. Gudin, C.; Daniel C. Cell fragility - the key problem of microalgae mass production in closed photo bioreactors. Bio resource Technology. 1991, 38, 145-151. 
7. Eibl, R.; Dieter, E. Design of bioreactors suitable for plant cell and tissue cultures. Phytochemistry Reviews, 2008, 7, 593-598.

8. Narendranathan, T.J. Designing fermentation equipment. Chemical engineer. 1986, 425, 23-31.

9. Martin, I.; Wendt, D.; Heberer, M.The role of bioreactors in tissue engineering. Trends in biotechnology. 2004, 22, 80-86.

10. Chen, H.C.; Hu Y.C.Bioreactors for tissue engineering. Biotechnology letters. 2006, 28, 1415-1423.

11. Singh, J.;Kaushik, S.; Biswas, S. Bioreactors-Technology \& Design Analysis. The Scitech J.2014, 1.

12. Duurkoop, A. Stainless steel reflects the quality of Applikon's bioreactors. Bioteknowledge (Applikon, Holland). 1992,12, 4-6.

13. Alaghlavi. Design of Fermenter and Kinetics.Bioprocess Engineering. 2013.

14. Nienow, A. W. Stirring and stirred-tank reactors. ChemieIngenieurTechnik.2014, 86, 2063-2074.

15. McNeil, B.; Harvey, L. eds. Practical fermentation technology. John Wiley \& Sons. 2008.

16. Raj, A.E.; Karanth, N.G. Fermentation technology and bioreactor design. Food Science and Technology New York Marcel dekkar. 2006, 148, 33.

17. Abbott, M.S.; Harvey, A.P.; Perez, G.V.; Theodorou, M.K. Biological processing in oscillatory baffled reactors: operation, advantages and potential. Interface focus. 2013, 3.1, 20120036.

18. Martín, M.; Montes, F.J.; Galán, M.A. On the contribution of the scales of mixing to the oxygen transfer in stirred tanks. Chemical Engineering Journal. 2008, 145, $232-$ 241.

19. Garcia-Ochoa, F.; Gomez, E. Bioreactor scale-up and oxygen transfer rate in microbial processes: an overview. Biotechnology advances. 2009, 27, 153-176.

20. Tang, Y.Q.; An, M.Z.; Zhong, Y.L.; Shigeru, M.; Wu, X.L.; Kida, K. Continuous ethanol fermentation from non-sulfuric acid-washed molasses using traditional stirred tank reactors and the flocculating yeast strain KF-7. Journal of Bioscience and Bioengineering. 2010, 109, 41-46.

21. Liu, H.S.; Hsien-Wen, H. Analysis of gas stripping during ethanol fermentation-I In a continuous stirred tank reactor. Chemical Engineering Science. 1990,45, 1289-1299.

22. Chisti, Y. Fermentation technology. Industrial Biotechnology, Sustainable Growth and Economic Success.2010, 149-171.

23. Ali, S.; Haq, I.U.; Qadeer, M.A.; Iqbal, J. Production of citric acid by Aspergillusniger using cane molasses in a stirred fermenter. Electronic Journal of Biotechnology.2002, 5, 19-20.

24. Vamanu, E. Studies regarding the production of probiotic biomass from Lactobacillus plantarum strains. Arh. Zootechn. 2009, 12, 92101.

25. Veera, U.P.; Kataria, K.L.; Joshi, J.B.Gas hold-up profiles in foaming liquids in bubble columns. Chemical Engineering Journal.2001, 84, 247-256.

26. Chisti, Y.; Moo-Young, M. Bioreactor design. Basic biotechnology. 2001, 151-72. 
27. Chisti, Y.; Moo-Young, M. Fermentation technology, Bioprocessing, Scaleup and. Biotechnology-The Science and the Business. 1999, 177.

28. Siegel, M.H.; Robinson, C.W. Application of airlift gas-liquid-solid reactors in biotechnology. Chemical Engineering Science. 1992, 47, 3215-3229.

29. Kantarci, N.; Borak, F.; Ulgen, K.O. Bubble column reactors. Process biochemistry. 2005, 40, 2263-2283.

30. Degaleesan, S.; Dudukovic, M.; Pan, Y. Experimental study of gas induced liquidflow structures in bubble columns, AIChE J.2001, 47, 913-1931.

31. Prakash, A.; Margaritis, A.; Li, H.; Bergougnou, M.A. Hydrodynamics and local heat transfer measurements in a bubble column with suspension of yeast. Biochemical Engineering Journal. 2001,9, 155-163.

32. Ogbonna, J.C.; Mashima, H.; Tanaka, H. Scale up of fuel ethanol production from sugar beet juice using loofa sponge immobilized bioreactor. Bioresource Technology. 2001, 76, 1-8.

33. Wilkinson, P.M.; Spek, A.P.; Van Dierendonck, L.L. Design parameters estimation for scale-up of high-pressure bubble columns. AIChE Journal.1992,38, 544-554.

34. Gibilaro, L.G. Fluidization-Dynamics. The formulation and application of a predictive theory for the fluidized state. Firth Publication-Butterworth-Heinemann. 2001.

35. Kwong, W. H. An improved simplified model predictive control algorithm and its application to a continuous fermenter. Brazilian Journal of Chemical Engineering. 2000, 17,143-161.

36. Wang, G.; Zhang, W.; Jacklin, C.; Freedman, D.; Eppstein, L.; Kadouri, A. Modified CelliGen-packed bed bioreactors for hybridoma cell cultures. Cytotechnology.1992, 9 , 41-49.

37. Siegel, M.H.; Robinson, C.W. Application of airlift gas-liquid-solid reactors in biotechnology. Chemical Engineering Science. 1992, 47, 3215-3229.

38. Chang, H.N.; Furusaki, S. Membrane bioreactors: present and prospects. In Bioreactor systems and effects Springer, Berlin, Heidelberg.1991, 27-64.

39. Mavituna, F.; Atkinson, B. Biochemical engineering and biotechnology handbook. No. 600.6 A85 1983. New York: Stockton press, 1991.

40. Lau, R.; Lee, P.H.V.; Chen, T. Mass transfer studies in shallow bubble column reactors. Chemical engineering and processing. Process intensification. 2012, 62, 18-25.

41. Lebrero, R.; Rodríguez, E.; Martin, M.; García-Encina, P.A.; Muñoz, R. H2S and VOCs abatement robustness in bio filters and air diffusion bioreactors: a comparative study. Water research.2010, 44, 3905-3914.

42. Garcia-Ochoa, F.; Gomez, E. Mass transfer coefficient in stirred tank reactors for xanthan gum solutions. Biochemical Engineering Journal. 1998, 1, 1-10.

43. Rolfe, P. Sensing in tissue bioreactors. Measurement Science and Technology. 2006, $17,578$.

44. Junker, B.H. Scale-up methodologies for Escherichia coli and yeast fermentation processes. Journal of bioscience and bioengineering, 2004, 97, 347-364.

45. Schmidt, F.R. Optimization and scale up of industrial fermentation processes. Applied microbiology and biotechnology. 2005, 68, 425-435. 
46. Stanbury, P.F.; Whitaker, A.; Hall, S.J. Principles of fermentation technology. Elsevier, 2013.

47. Blakebrough, N. Fundamentals of fermenter design. Pure and Applied Chemistry. 1973, 36, 305-316.

48. Singhal, G.; Verma, V.; Bhagyawant, S.S.; Srivastava, N. Fermentation Technology Prospecting on Bioreactors/Fermenters: Design and Types. Principles and Applications of Fermentation Technology, 2018, 65-83.

49. Doran, P.M. Bioprocess engineering principles. Elsevier, 1995. 